\title{
Concurrent photocatalytic hydrogen production and organic degradation by a composite catalyst film in a two chamber photo-reactor
}

\author{
Xi Wang ${ }^{1,2}$, Xiao-yan $\mathrm{Li}^{1, *}$ \\ ${ }^{1}$ Environmental Engineering Research Centre, Department of Civil Engineering, The University of Hong \\ Kong, Pokfulam Road, Hong Kong, China \\ ${ }^{2}$ School of Chemistry and Environment, South China Normal University, Guangzhou, Guangdong, China \\ (*Corresponding author: phone: 852-28592659; fax: 852-28595337; e-mail: xlia@hkucc.hku.hk)
}

\begin{abstract}
A novel visible light-driven photocatalyst film, $\mathrm{MoS}_{2} / \mathrm{Ag} / \mathrm{TiO}_{2}$, was synthesized on a glass-fiber membrane. The composite catalyst film had a multi-layer structure with Ag as nanoconjunctions between the $\mathrm{MoS}_{2}$ and $\mathrm{TiO}_{2}$ layers. The catalyst film performed well for both photocatalytic hydrogen production and organic degradation in a two-chamber photo-reactor under either solar or visible light. Hydrogen was produced in the cathode side chamber while the model organic was decomposed in the anode side chamber. The specific hydrogen production rate went through a maximum of $85 \mathrm{mmol} / \mathrm{m}^{2}-\mathrm{h}$ with an energy conversion efficiency of $0.85 \%$, while the maximum specific organic carbon removal for formic acid under solar light reached $1520 \mathrm{mg} / \mathrm{m}^{2}$-h. It is apparent that $\mathrm{Ag}$ between the $\mathrm{TiO}_{2}$ and $\mathrm{MoS}_{2}$ layers allowed the transfer of photo-excited electrons via $\mathrm{TiO}_{2} \rightarrow \mathrm{Ag} \rightarrow \mathrm{MoS}_{2}$ for organic degradation and $\mathrm{H}^{+}$reduction (e.g. hydrogen evolution) in two different chambers.
\end{abstract}

\section{Keywords}

Hydrogen production, $\mathrm{MoS}_{2}$, organic degradation, photocatalyst, solar energy

\section{INTRODUCTION}

Hydrogen is one of the most promising clean and renewable energy carriers in future. Photocatalytic hydrogen generation from water is an attractive and environmentally-friendly method to harvest the solar energy (Lee et al., 2007; Maeda et al., 2006). The photocatalytic process also has attracted significant research attentions as a potential energy-saving technology for effective pollution removal in water and wastewater treatment (Balasubramanian et al., 2004; Carp et al., 2004; Chatterjee and Dasgupta, 2005).

In recent years, $\mathrm{MoS}_{2}$ has been shown as a promising photocatalyst that responds to visible light. $\mathrm{MoS}_{2}$ possesses a significant advantage over $\mathrm{TiO}_{2}$ which is a UV-driven photocatalyst, i.e. the band gap of $\mathrm{MoS}_{2}$ is small enough to allow absorption of visible light. The $\mathrm{MoS}_{2} / \mathrm{TiO}_{2}$ catalyst powders have shown their effectiveness in degradation of organic chemical pollutants, such as methylene orange (Hu et al., 2010), 4-chlorophenol (Ho et al., 2004) and phenol (Pourabbas and Jamshidi, 2008). Hence, $\mathrm{MoS}_{2}$-based materials can be used for development of visible light-driven catalyst for both photocatalytic hydrogen generation and organic degradation. In the present study, a composite $\mathrm{MoS}_{2} / \mathrm{Ag} / \mathrm{TiO}_{2}$ photocatalyst film was synthesized with Ag as the nanojunction between the $\mathrm{MoS}_{2}$ and $\mathrm{TiO}_{2}$ layers. The photocatalyst film was placed between two chambers in a photo-reactor to achieve hydrogen production together with organic degradation. 


\section{MATERIALS AND METHODS}

\section{Preparation of $\mathrm{MoS}_{2}$ nanopartiles}

One hundred $\mathrm{mL} 1.0 \mathrm{~mol} / \mathrm{L} \mathrm{HCl}$ solution was placed in a flask and then the solution was heated at $90{ }^{\circ} \mathrm{C}$ with continuous stirring. Then, $0.88 \mathrm{~g}\left(\mathrm{NH}_{4}\right)_{6} \mathrm{Mo}_{7} \mathrm{O}_{24} \cdot 4 \mathrm{H}_{2} \mathrm{O}$ and $2.64 \mathrm{~g} \mathrm{Na} \mathrm{Na}_{2} \mathrm{~S} \cdot 9 \mathrm{H}_{2} \mathrm{O}$ were added into the flask. After half an hour, $0.7 \mathrm{~g} \mathrm{NH} \mathrm{N}_{2} \mathrm{OH} \cdot \mathrm{HCl}$ was added with vigorous stirring. The reaction was kept at $90^{\circ} \mathrm{C}$ for 6 hrs. During the heating process, the deep red color of the precursor turned to the black color of $\mathrm{MoS}_{2}$ as expected (Tian et al., 2005). The solid precipitates were separated from the solution by centrifugation. The final product was washed several times with DI water to remove residual reactants. The solids were dried in air to obtain the final $\mathrm{MoS}_{2}$ powder.

\section{Characterization of $\mathrm{MoS}_{2}$ photocatalyst}

The size distribution of the $\mathrm{MoS}_{2}$ catalyst particles was measured by a laser diffraction particle size analyzer (Delsa ${ }^{\mathrm{TM}}$ Nano, Beckman Coulter). The crystalline phases and structural features of $\mathrm{MoS}_{2}$ were analyzed by an X-ray diffraction (XRD) system (D8 Advance, Burker AXS) with the $\mathrm{Cu} \mathrm{K} \alpha$ irradiation from 10 to 90 degrees. The morphology of the $\mathrm{MoS}_{2}$ particles was examined by SEM and TEM.

\section{$\mathrm{MoS}_{2} / \mathrm{Ag} / \mathrm{TiO}_{2}$ film}

Glass fiber (GF) membrane was used as the base material for immobilization of the photocatalysts. The membrane was cut into circles (area $=7 \mathrm{~cm}^{2}$ ), and Nafion was employed to affix the catalyst film on the membrane surface. The $\mathrm{MoS}_{2}$ nanoparticles (50 mg) were mixed with Nafion solution and applied to the membrane. Ag was then deposited on the $\mathrm{MoS}_{2}$ surface by in-situ photo-deposition. Finally, a Nafion $\mathrm{TiO}_{2}$ (Degussa, P-25) paste was applied over the $\mathrm{MoS}_{2}$ layer on the membrane, and the $\mathrm{TiO}_{2}$ loading ratio varied from 10 to $200 \%$ of the amount of $\mathrm{MoS}_{2}$ layer on the GF membrane.

The performance of different catalyst films were tested in a photo-reactor with two chambers. The photocatalyst film was placed between the two chambers to separate the chambers. A 300W Xe lamp setup (PLS-SXE Xe light source, Trustech) was used as either a visible light source (light intensity $\left.\sim 70 \mathrm{~mW} / \mathrm{cm}^{2}\right)$ with a cutoff $(\lambda<420 \mathrm{~nm}$ ) filter or a combined UV and visible light source light intensity $\sim 86 \mathrm{~mW} / \mathrm{cm}^{2}$ ) without the cutoff filter. Light irradiation was applied on the $\mathrm{TiO}_{2}$ side of the catalyst film from the anode side chamber. The anode chamber was filled with $10 \%$ formic acid solution as the model organic at $\mathrm{pH}=7$. The cathode side chamber was filled with water containing $0.4 \mathrm{M} \mathrm{Na}_{2} \mathrm{SO}_{4}$ at $\mathrm{pH}=1.0$ for hydrogen production. The gas produced during the photo-tests was collected, and the $\mathrm{H}_{2}$ and $\mathrm{CO}_{2}$ contents were analyzed by a gas chromatograph (GC HP5890 Series II, Hewlett Packard). The reactivity of the photocatalyst was evaluated in terms of the specific $\mathrm{H}_{2}$ production rate $(R)$ and the corresponding energy conversion efficiency $(\eta)$ using the following equations.

$$
\begin{aligned}
& R_{A}=\frac{m_{H_{2}}}{A t}, \text { and } \\
& \eta=\frac{R_{A} \Delta H_{C}}{I}
\end{aligned}
$$

where $m_{\mathrm{H}_{2}}$ is the moles of $\mathrm{H}_{2}$ production measured, $t$ is the duration of photo-reaction, $W$ is the weight of catalyst in reactor, $\Delta H_{c}$ is the combustion value of $\mathrm{H}_{2}(286 \mathrm{~kJ} / \mathrm{mol})$, and $A$ and $I$ is specify the area $\left(7 \mathrm{~cm}^{2}\right)$ and intensity of the light irradiation, respectively. 


\section{RESULTS AND DISCUSSION}

\section{Characterization of the $\mathrm{MoS}_{2}$ catalysts}

Figure 1 shows the particle size distribution of the $\mathrm{MoS}_{2}$ catalyst. The catalyst powder ranged in size from 230 to $340 \mathrm{~nm}$ with a number based mean size of about $275 \mathrm{~nm}$. The TEM images in Figure 2 further reveal that the photocatalysts are nano-particles with a size of around $80 \mathrm{~nm}$ for the primary particles. The $\mathrm{MoS}_{2}$ catalyst displaced a good crystallization as shown by its XRD pattern (Figure 3).

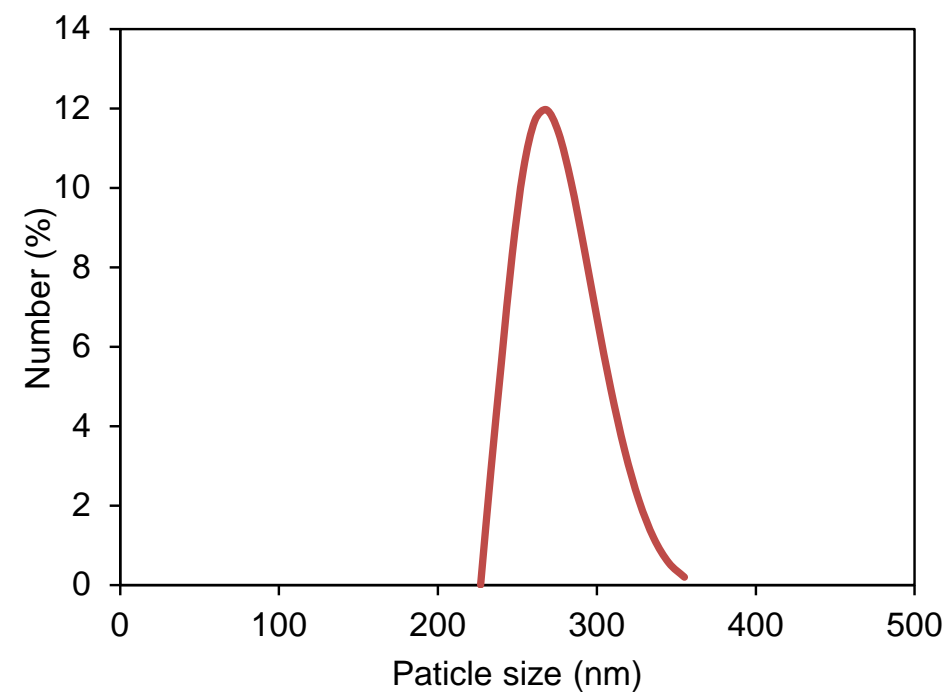

Figure 1. The particle size distribution of $\mathrm{MoS}_{2}$ catalysts.

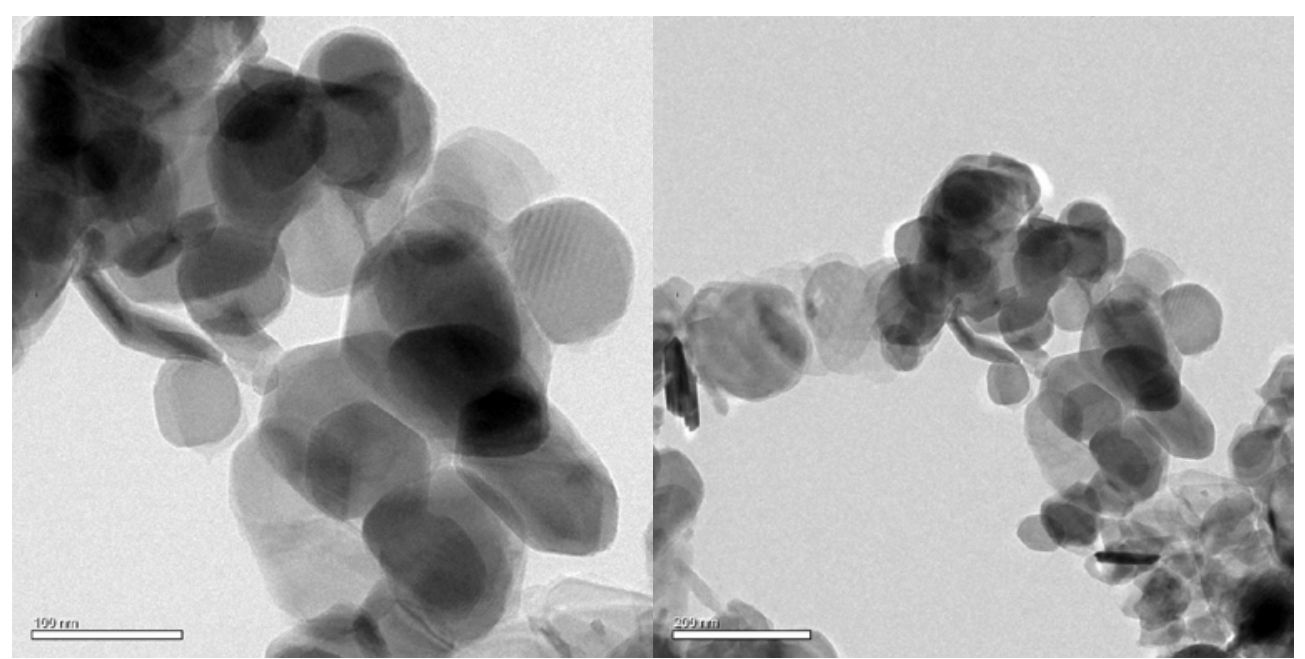

Figure 2. TEM images of $\mathrm{MoS}_{2}$ nanoparticles.

Figure 4 shows the diffuse reflectance spectrum of the $\mathrm{MoS}_{2}$ catalyst. Its absorption edge is in the visible light range at about $790 \mathrm{~nm}$, corresponding to a band gap of $1.7 \mathrm{eV}$. This suggests the visible light-driven feature of $\mathrm{MoS}_{2}$ for photocatalytic reactions under visible light. 


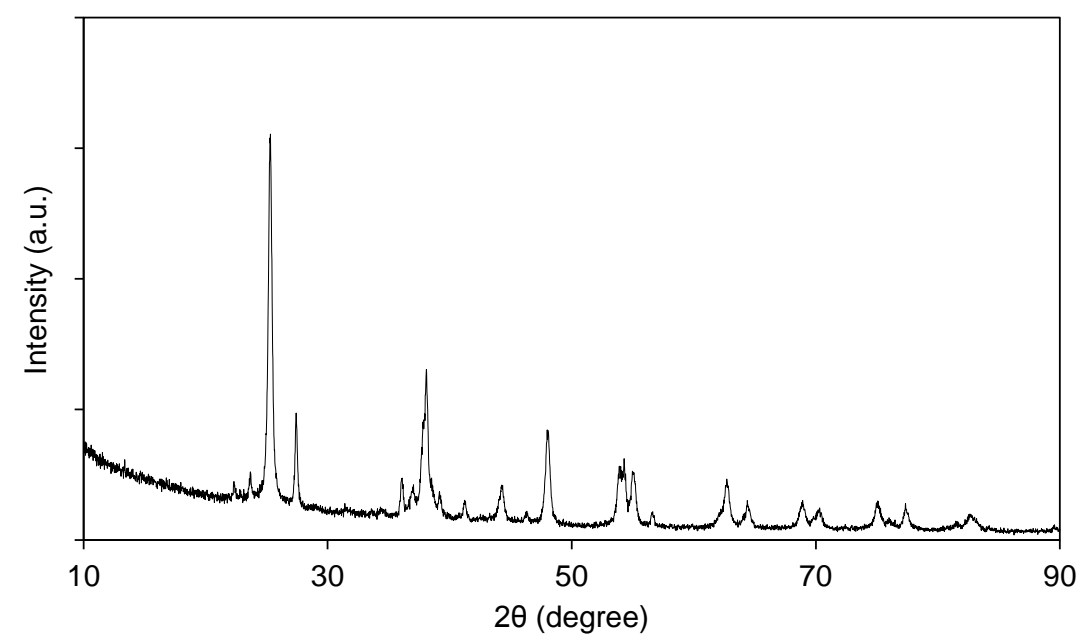

Figure 3. XRD pattern of $\mathrm{MoS}_{2}$.

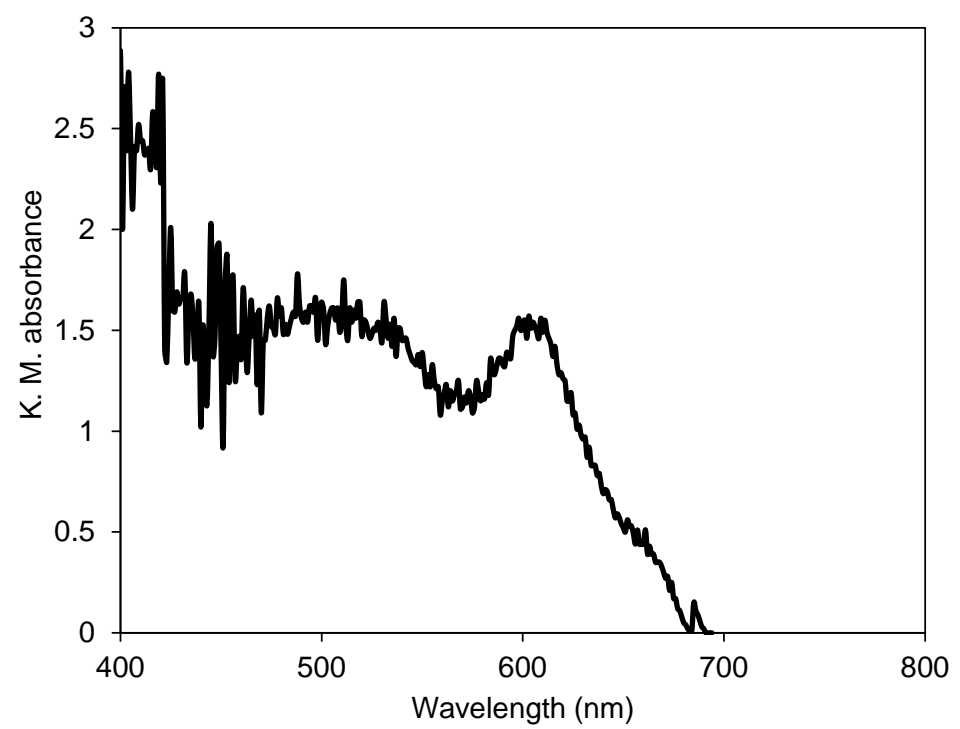

Figure 4. Diffuse reflectance spectrum of the $\mathrm{MoS}_{2}$ photocatalyst.

\section{$\mathrm{MoS}_{2} / \mathrm{Ag} / \mathrm{TiO}_{2}$ photocatalyst film for hydrogen production}

Results of photocatalytic $\mathrm{H}_{2}$ generation and organic degradation $\left(\mathrm{CO}_{2}\right.$ production) obtained with the $\mathrm{MoS}_{2} / \mathrm{Ag} / \mathrm{TiO}_{2}$ film irradiated by the simulated solar light are shown in Figure 5A, where the gas production rates are plotted as a function of the $\mathrm{TiO}_{2}$ loading ratio.

With $10 \%$ formic acid in the anode side chamber, the bare $\mathrm{MoS}_{2}$ was not capable of photocatalytic hydrogen production. Coating of the outer $\mathrm{TiO}_{2}$ layer could significantly enhance the photo-reactivity of the $\mathrm{MoS}_{2}$ catalyst for both $\mathrm{H}_{2}$ production and organic degradation (Figure 5). As the $\mathrm{TiO}_{2}$ loading ratio increased to $160 \%$ of the $\mathrm{MoS}_{2}$ layer, the area-based specific $\mathrm{H}_{2}$ and $\mathrm{CO}_{2}$ production rates increased to the maximum values of 88 and $85 \mathrm{mmol} / \mathrm{m}^{2}-\mathrm{h}$ in the respective cathode and anode chambers under solar light (Figure 5A) with an energy conversion efficiency of $0.85 \%$ and a quantum yield of $12 \%$. Under only the visible light, similar results for the effect of $\mathrm{TiO}_{2}$ coating were obtained with somewhat lower specific $\mathrm{H}_{2}$ and $\mathrm{CO}_{2}$ production rates (Figure 5B).

Based on the amount of $\mathrm{CO}_{2}$ production from the anode side chamber, the corresponding specific organic degradation rate in terms of the total organic carbon (TOC) removal was estimated (Figure 6). The maximum specific TOC removal for formic acid under solar light was 1520 $\mathrm{mg} / \mathrm{m}^{2}-\mathrm{h}$ at a $\mathrm{TiO}_{2}$ load of $160 \%$. 

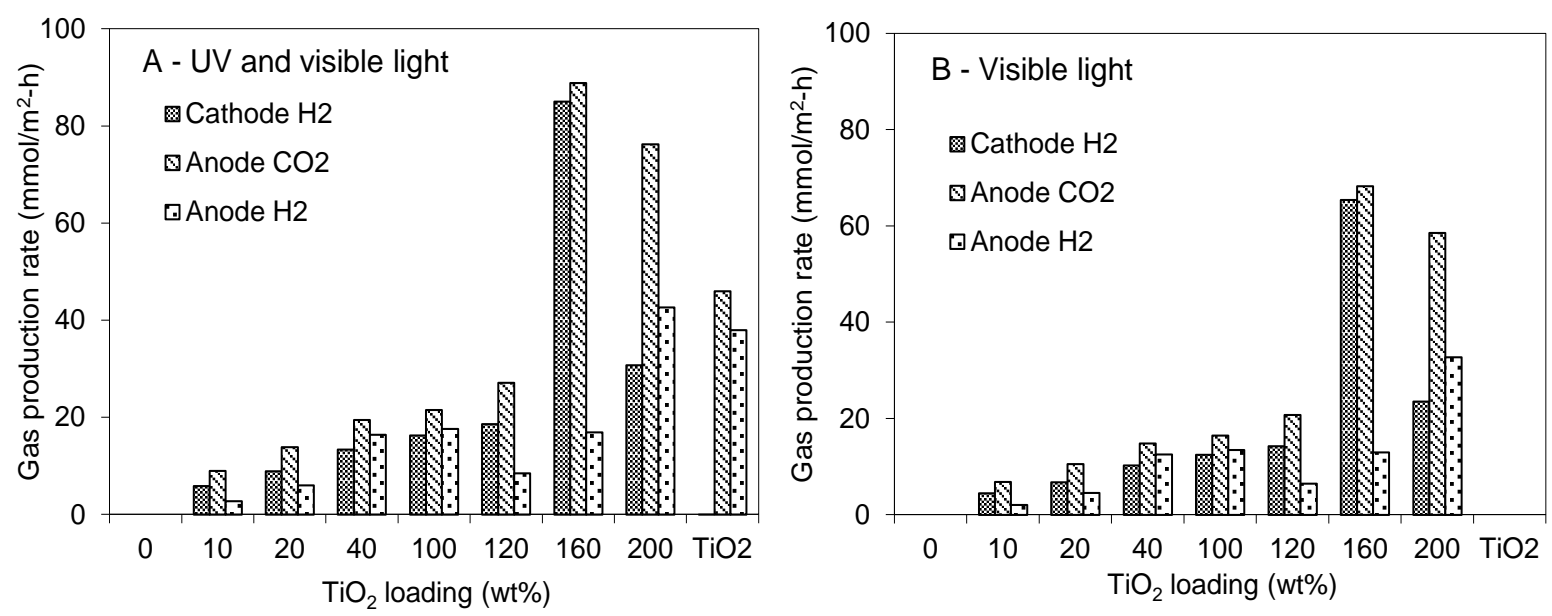

Figure 5. Hydrogen and carbon dioxide production rates for the $\mathrm{MoS}_{2} / \mathrm{Ag} / \mathrm{TiO}_{2}$ catalyst films with different $\mathrm{TiO}_{2}$ loading ratios.

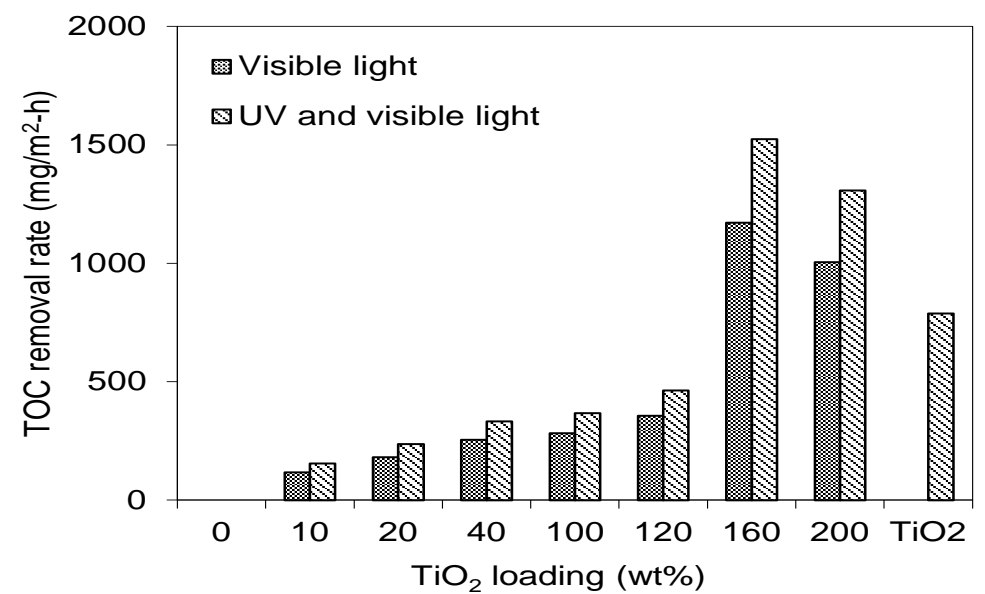

Figure 6. Photocatalytic TOC removal rates by the $\mathrm{MoS}_{2} / \mathrm{Ag} / \mathrm{TiO}_{2}$ photocatalyst film with different $\mathrm{TiO}_{2}$ loading ratios.

It is apparent that the composite $\mathrm{MoS}_{2} / \mathrm{Ag} / \mathrm{TiO}_{2}$ multi-layer structure rendered a synergetic effect of the catalyst materials for photocatalytic $\mathrm{H}_{2}$ generation and organic degradation. The more photosensitive $\mathrm{MoS}_{2}$ base layer ensured the visible absorbance and induced the reactivity of the photocatalyst. The use of the $\mathrm{TiO}_{2}$ outer layer would help suppress the recombination of electron/hole pairs formed on the $\mathrm{MoS}_{2}$ inner layer, making the electrons more available for $\mathrm{H}^{+}$ reduction and $\mathrm{H}_{2}$ evolution on the cathode side (Best and Dunstan, 2009; Cao and Banin, 2000). As the electrons transferring from $\mathrm{TiO}_{2}$ to $\mathrm{MoS}_{2}$, the holes left on the VB of $\mathrm{TiO}_{2}$ would render a considerable oxidation capability for organic degradation on the anode side. Nonetheless, further increase in the $\mathrm{TiO}_{2}$ loading ratio did not result in any increase in $\mathrm{H}_{2}$ generation on the cathode side and organic degradation on the anode side (Figure 5).

The photocatalytic mechanism of the $\mathrm{MoS}_{2} / \mathrm{Ag} / \mathrm{TiO}_{2}$ multiple-layer system may be expected as follows. During the photocatalytic process, electron transfer via a direction of $\mathrm{TiO}_{2} \rightarrow \mathrm{Ag} \rightarrow \mathrm{MoS}_{2}$ should occur as a result of the excitation of both $\mathrm{TiO}_{2}$ and $\mathrm{MoS}_{2}$. The electron transfer would suppress the recombination of the photo-generated electron-hole pairs. Accordingly, the oxidation and reduction capabilities of the photocatalyst film were greatly improved, resulting in hydrogen production and organic degradation in two chambers on different sides of the catalyst film. 


\section{CONCLUSIONS}

As an alternative and more environmentally friendly photocatalyst, the $\mathrm{MoS}_{2} / \mathrm{Ag} / \mathrm{TiO}_{2}$ multi-layer catalyst film was synthesized on the glass fiber membrane with Ag as the nanojunctions between the two catalyst layers. The catalyst film was used in a two-chambered photo-reactor to achieve separate photocatalytic hydrogen production and organic degradation. The composite catalyst film performed well for concurrent photocatalytic hydrogen production and organic degradation in the photo-reactors under either solar light or visible light irradiation. The specific hydrogen production rate went through a maximum of $85 \mathrm{mmol} / \mathrm{m}^{2}-\mathrm{h}$ with an energy conversion efficiency of $0.85 \%$, while the corresponding specific TOC removal for formic acid under solar light reached $1520 \mathrm{mg} / \mathrm{m}^{2}$-h.

\section{ACKNOWLEDGMENTS}

This study was supported by SRT and URC funding from The University of Hong Kong and a Special Equipment Grant SEG_HKU10 from the University Grants Committee (UGC) of the Hong Kong SAR Government. The technical assistance of Mr. Keith C.H. Wong is highly appreciated.

\section{REFERENCES}

Balasubramanian, G., Dionysiou, D.D., Suidan, M.T., Baudin, I. and Laîné, J.M. (2004). Evaluating the activities of immobilized $\mathrm{TiO}_{2}$ powder films for the photocatalytic degradation of organic contaminants in water. Applied Catalysis B: Environmental, 47(2), 73-84.

Best, J.P. and Dunstan, D.E. (2009). Nanotechnology for photolytic hydrogen production: colloidal anodic oxidation. International Journal of Hydrogen Energy, 34(18), 7562-7578.

Cao, Y.W. and Banin, U. (2000). Growth and properties of semiconductor core/shell nanocrystals with InAs cores. Journal of the American Chemical Society, 122(40), 9692-9702.

Carp, O., Huisman, C.L. and Reller, A. (2004). Photoinduced reactivity of titanium dioxide. Progress in Solid State Chemistry, 32(1-2), 33-177.

Chatterjee, D. and Dasgupta, S. (2005). Visible light induced photocatalytic degradation of organic pollutants. Journal of Photochemistry and Photobiology C-Photochemistry Reviews, 6(2-3), 186-205.

Ho, W.K., Yu, J.C., Lin, J., Yu, J.G. and Li, P.S. (2004). Preparation and photocatalytic behavior of $\mathrm{MoS}_{2}$ and $\mathrm{WS}_{2}$ nanocluster sensitized $\mathrm{TiO}_{2}$. Langmuir, 20(14), 5865-5869.

Hu, K.H., Hu, X.G., Xu, Y.F. and Sun, J.D. (2010). Synthesis of nano- $\mathrm{MoS}_{2} / \mathrm{TiO}_{2}$ composite and its catalytic degradation effect on methyl orange. Journal of Materials Science, 45(10), 2640-2648.

Lee, Y., Terashima, H., Shimodaira, Y., Teramura, K., Hara, M., Kobayashi, H., Domen, K. and Yashima, M. (2007). Zinc germanium oxynitride as a photocatalyst for overall water splitting under visible light. Journal of Physical Chemistry C, 111(2), 1042-1048.

Maeda, K., Teramura, K., Lu, D.L., Takata, T., Saito, N., Inoue, Y. and Domen, K. (2006). Photocatalyst releasing hydrogen from water - enhancing catalytic performance holds promise for hydrogen production by water splitting in sunlight. Nature, 440(7082), 295.

Pourabbas, B. and Jamshidi, B. (2008). Preparation of $\mathrm{MoS}_{2}$ nanoparticles by a modified hydrothermal method and the photo-catalytic activity of $\mathrm{MoS}_{2} / \mathrm{TiO}_{2}$ hybrids in photo-oxidation of phenol. Chemical Engineering Journal, 138(1-3), 55-62.

Tian, Y.M., Zhao, J.Z., Fu, W.Y., Liu, Y.H., Zhu, Y.Z. and Wang, Z.C. (2005). A facile route to synthesis Of $\mathrm{MoS}_{2}$ nanorods. Materials Letters, 59(27), 3452-3455.

Wang, X., Shih, K. and Li, X.Y. (2010). Photocatalytic hydrogen generation from water under visible light using core/shell nano-catalysts. Water Science and Technology, 61(9), 2303-2308. 